\title{
An Active Region Model for Capturing Fractal Flow Patterns in Unsaturated Soils: Model Development
}

\author{
H.H. Liu ${ }^{\mathrm{a}}$, R. Zhang ${ }^{\mathrm{b}, \mathrm{c}}$ and G. S. Bodvarsson ${ }^{\mathrm{a}}$ \\ ${ }^{a}$ Earth Sciences Division, Lawrence Berkeley National Laboratory, Berkeley, California \\ ${ }^{b}$ Department of Renewable Resources, University of Wyoming, Laramie, Wyoming \\ ${ }^{\mathrm{c}}$ School of Environmental Science and Engineering, Sun Yat-Sen (ZhongShan) Unversity, \\ Guangzhou, China
}

* Corresponding author (email: hhliu@lbl.gov) 


\section{Abstract}

2 Preferential flow commonly observed in unsaturated soils allows rapid movement of

3. solute from the soil surface or vadose zone to the groundwater, bypassing a significant

4 volume of unsaturated soil and increasing the risk of groundwater contamination. A

5 variety of evidence indicates that complex preferential patterns observed from fields are

6 fractals. In this study, we developed a relatively simple active region model to

7 incorporate the fractal flow pattern into the continuum approach. In the model, the flow

8 domain is divided into active and inactive regions. Flow occurs preferentially in the

9 active region (characterized by fractals), and inactive region is simply bypassed. A new

10 constitutive relationship (the portion of the active region as a function of saturation) was

11 derived. The validity of the proposed model is demonstrated by the consistency between

12 field observations and the new constitutive relationship.

13

14

15

16

17

18

19

20

21

22

23

24

25

26

27

28

29

30

31

Key words: Preferential flow, Fractal, Constitutive relations, Vadose zone hydrology.

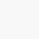

(6)

17

(

(

2


1

2

\section{Introduction}

It has been recognized that preferential flow is common for natural unsaturated soils. Preferential flow results in that liquid water propagates quickly to significant depths while bypassing large portions of the involved soils, and solute travel times from the contamination source (located in soil surface or vadose zone) to groundwater are shorter than a prior expected (e.g., Flury et al., 1994). Because of the important effects of this flow process on groundwater contamination, preferential flow has been a major research area in the vadose zone hydrology community for many years (Simunek et al., 2003).

Field-scale preferential flow is caused by a number of well-known mechanisms. First, under flooding and/or high-infiltration-rate conditions, water can flow very quickly along macropores (such as cracks and fissures) in structured soils (Beven and Germann, 1982; Bouma and Dekker, 1991; Flury et al., 1994; Öhrström et al., 2002). Second, because of the high nonlinearity of the unsaturated flow process, an infiltrating water front can become unstable and split into "fingers" even for relatively homogeneous, structureless soils (Hillel, 1987; Glass et al., 1988; de Rooij 2000; Wang et al., 2003). Third, the spatial variability of soil properties also causes highly non-uniform flow patterns corresponding to preferential flow paths (e.g., Vogel et al., 2000). At the field scale, a combination of these three mechanisms likely contributes to the observed preferential flow processes, although one or two of them may be dominant mechanisms for a given testing site. For example, Olsson et al. (2002) reported that dye-stained preferential pathways were not only restricted to macropores in the topsoil, but also to fingers resulting from wetting front instability. 
1 Because of its complexity, preferential flow and the associated transport are probably

2 the most frustrating processes in terms of hampering accurate predictions of contaminant

3 transport in the vadose zone (Simunek et al., 2003). Recently, though, significant effort

4 has been made in developing a variety of approaches to deal with preferential flow and

5 transport. These approaches mainly fall into one of two categories: the continuum

6 approach and the discrete approach. An excellent review of the continuum-approach-

7 based models was recently given by Simunek et al. (2003) for structured (macropore)

8 soils. In the continuum approach, connected macropores and soil matrix are viewed as

9 two or more overlapped interacting continua. In other words, at a "point," two or more

10 continua are considered to co-exist. In this case, the continuum mechanics formulations

11 can be used to describe flow and transport in each continuum. Coupling of processes

12 between different continua is determined by their interaction mechanisms at a subgrid

13 scale. The similar approaches have also been used in modeling water flow and transport

14 in fractured rock (e.g., Doughty, 1999). Because the continuum approach is relatively

15 simple and straightforward to implement, it is preferred for most applications

16 encountered in practice. However, a major limitation of the currently available continuum

17 models is that they were mainly developed for dealing with the effects of macropores for

18 structured soils, and are unable to capture preferential flow paths resulting from fingering

19 and the spatial variability of soil properties.

20 In contrast to the continuum models, a number of researchers have suggested using

21 discrete modeling approaches, such as the diffusion limited aggregation (DLA) and

22 percolation-based approaches (e.g., Ewing and Berkowitz. 2001; Glass 1993; Flury and

23 Flühler, 1995). Roughly speaking, these approaches treat soil water body as discrete 
1 "particles" or "packages" whose movement is controlled by some simple rules to

2 generate growth patterns with rich structures (fractals). These approaches have been

3 successfully used to represent relatively small-scale observations. However, use of these

4 discrete approaches is quite limited for large-scale applications (Liu et al., 2003).

5 Furthermore, completely satisfactory theories underlying these approaches are still

6 missing, and some critical steps in these approaches are somewhat arbitrary (Meakin and

7 Tolman, 1989; Ewing and Berkowitz, 2001).

8 Obviously, an ideal approach to deal with preferential flow should be able to combine

9 the advantages of the continuum and discrete approaches. The objective of this study is to

10 develop an active region model that represents an effort toward developing an approach

11 of this kind by incorporating fractal flow patterns (often observed in the field and

12 simulated by discrete approaches) into the continuum approach. In the next section, we

13 will discuss field evidence of fractal flow patterns in unsaturated soils. Section 3 will

14 present details of the active region model and its relation to a fractal flow pattern. Section

154 will demonstrate the consistency between the active region model and the relevant field

16 observations.

17 2. Fractal flow patterns and preferential flow process

18 Fractals have been shown to provide a common language for describing many 19 different natural and social phenomena (Mandelbrot, 1982). While a vast literature exists 20 on the validity of the fractal concept for a great number of fields, fractals have been 21 found to be useful for representing many spatial distributions in subsurface hydrology, 22 including soil particle size distribution, roughness of fracture surface, distribution of 23 permeability in heterogeneous formations, and large-scale solute dispersion processes 
1 (e.g., Tyler and Wheatcraft, 1990; Rieu and Sposito, 1991a, b; Perfect et al., 1996;

2 Neuman, 1990, 1994; Molz and Boman, 1993; Molz et al., 1997).

3 Recent studies have also suggested that complex preferential flow patterns in

4 unsaturated systems can be characterized by fractals. Hatano and Booltink (1992) may be

5 the first authors to report in the vadose zone hydrology community that the geometry of

6 dyed flow patterns in two-dimensional images of soil profiles could be characterized by

7 fractals. Flury and Flühler (1995) indicated that solute leaching patterns, observed from

8 three field plots consisting of an unsaturated loamy soil, could be well represented by a

9 diffusion-limited aggregation (DLA) model (Witten and Sander, 1981), although the

10 relation between DLA parameters and soil hydraulic properties is still an unresolved

11 issue. The DLA model is a conceptual model describing the process where solid particles

12 irreversibly attach to each other and form aggregates and the process is limited by

13 diffusion. It has been documented that DLA generates fractal patterns (e.g., Feder, 1988;

14 Flury and Flühler, 1995). The observation of Flury and Flühler (1995) is further

15 supported by Persson et al. (2001), who used dye-infiltration data to investigate field

16 pathways of water and solutes under unsaturated conditions. Persson et al. (2001) showed

17 that field observations are well described by the DLA model. Furthermore, they

18 demonstrated that observed mean power spectrum for dye penetration of a field plot

19 displays a typical power-law relationship, another important indication of fractal flow

20 behavior. Öhrström et al. $(2002 ; 2004)$ also noticed that the dye penetrations for two test

21 sites (corresponding to a clayey and a sandy soil) are characterized by power-law mean

22 power spectrum. Olsson et al. (2002) reported that a field observation of dyed flow

23 pattern in an unsaturated test site is characterized by multi-fractals. This finding is 
1 consistent with that in many cases spatial distributions of hydraulic conductivity are

2 multi-fractals (Liu and Molz, 1997).

3 Related to preferential flow in unsaturated soils, fractal flow patterns have often been

4 observed in other unsaturated and multi-phase flow systems. Glass (1993) first showed

5 that unsaturated flow in a single vertical fracture is characterized by gravity-driven

6 fingers, and the resulting flow patterns could be modeled by an invasion-percolation

7 approach (Wilkinson and Wilemsen, 1983). Again, percolation-based models generate

8 fractal clustering patterns (Stauffer and Aharony, 2001). Viscous fingering in porous

9 media has been experimentally shown to be fractal (Feder, 1988). (The problem of

10 viscous fingering in porous media is of central importance in oil recovery.) Smith and

11 Zhang (2001) also reported that DNAPL fingering in water saturated porous media,

12 observed from sandbox experiments, is fractal. Liu et al. (2003) demonstrated that a

13 spatial distribution of fractures with mineral coatings is also fractal, while fracture

14 coating is roughly a signature of water flow paths.

15 3. An active region model

16 As discussed in Section 2, highly non-uniform (preferential) flow patterns in 17 unsaturated soil (and other unsaturated and multi-phase flow systems) are fractal. The 18 success of discrete approaches in modeling preferential flow largely relies on their capabilities for generating fractal patterns. Therefore, it is critical to incorporate fractal 20 flow patterns within a continuum model for it to capture preferential flow behavior. To 21 do so, we develop, in this sudy, an active region model. The main idea behind the active region model is that flow domain can be divided into active and inactive regions. Flow 
1 occurs preferentially in the active region (characterized by fractals) and inactive

2 (immobile) region is simply bypassed.

\section{$4 \quad 3.1$ Fractal dimension}

5 The key parameter for a fractal pattern is fractal dimension. Fractal dimension, $d_{f}$, is 6 generally a noninteger and less than the corresponding Euclidean (topological) dimension

7 of a space, D. Different kinds of definitions for fractal dimension exist (e.g., similarity

8 dimension, Hausdorff dimension, and box dimension), although they provide very close

9 fractal dimension values for practical applications (Feder, 1988). The most

10 straightforward definition is the so-called box dimension, based on a simple "box-

11 counting" procedure. This dimension is determined from Equation (1) (below) by

12 counting the number $(N)$ of "boxes" (e.g., line segments, squares and cubes for one-,

13 two-, and three-dimensional problems, respectively) needed to cover a spatial pattern, as

14 a function of box size $(l)$ (e.g., Feder, 1988):

15

16

$$
N(l)=\left(\frac{L}{l}\right)^{d} f
$$

where $L$ refers to the size of the entire spatial domain under consideration. Fig. 1 shows a

19 box-counting procedure for a spatial pattern with $\mathrm{d}_{\mathrm{f}}=1.6$, in a two-dimensional domain

20 with size L (Yamamoto et al., 1993).

21 Obviously, if a spatial pattern is uniformly distributed in space, the fractal dimension 22 will be identical to the corresponding Euclidean dimension. In this case, the number of 23 boxes that cover the pattern, $\mathrm{N}^{*}$, and the box size $l$ have the following relation 


$$
N *(l)=\left(\frac{L}{l}\right)^{D}
$$

3 A fractal pattern exhibits similarity at different scales. When $d_{f}<D$, the corresponding

4 pattern does not fill the whole space, but only part of it (Fig. 1).

\section{$5 \quad 3.2$ Characterization of fractal flow patterns}

6 To incorporate the effects of fractal flow patterns, we need to develop a simple scheme

7 to characterize these patterns in terms of parameters relevant to water flow processes.

8 Consider Fig. 1(a) to be a gridblock containing an active flow region and the

9 corresponding flow pattern to be fractal. In this case, only a portion of the medium within

10 a gridblock contributes to water flow (Fig. 1). This is conceptually consistent with the

11 preferential flow process. Note that in Fig. 1, a box is shadowed if it covers the active

12 flow region.

13 Combining Equations (1) and (2) yields

14

$15 \quad[N(l)]^{1 / d_{f}}=[N *(l)]^{1 / D}$

16

17 The average active water saturation $\left(S_{\mathrm{e}}{ }^{*}\right)$ for the whole gridblock (Fig. 1a) is determined

18 to be

19

$20 \quad S_{e}^{*}=\frac{V}{l^{D} \phi N *(l)}$

21 where $\mathrm{V}$ is the total water volume (excluding residual water) in the active region for the 
1 gridblock (Fig. 1a), and $\phi$ is the effective porosity (corresponding to saturated water

2 content excluding residual water content). Similarly, the average active water saturation

$3 \quad\left(\mathrm{~S}_{\mathrm{b}}{ }^{*}\right)$ for shadowed boxes with size of $l$ is

4

$5 \quad S_{b} *=\frac{V}{l^{D} \phi N(l)}$

6 From Fig. 1, it is obvious that there exists a box size $l_{1}<\mathrm{L}$ satisfying:

$7 \quad \frac{V}{l_{1}^{D} \phi}=1$

8 Based on Equations (3)-(6), the average saturation for shadowed boxes with size $l_{1}, S_{\mathrm{b} 1}$, $9 \quad$ can be expressed by

$10 \quad S_{b 1} *=\left(S_{e}^{*}\right)^{\frac{d f}{D}}$

11 Because a fractal is similar at different scales, the procedure to derive Equation (7)

12 from a gridblock with size $\mathrm{L}$ can be applied to shadowed boxes with the smaller size of $l_{l}$.

13 In this case, for a given box size smaller than $l_{l}$, the number of shadowed boxes will be 14 counted as an average number for those within the (previously shadowed) boxes with a 15 size of $l_{1}$. Again, we can find a box size $l_{2}<l_{1}$ to obtain a saturation relation:

$16 \quad S_{b 2} *=\left(S_{b 1} *\right) \frac{d_{f}}{D}=\left(S_{e}^{*}\right)^{\left(\frac{d_{f}}{D}\right)^{2}}$

18 The procedure to obtain Equation (8) can be continued until it reaches an iteration level, $19 \mathrm{n}^{*}$, at which all the shadowed boxes with a size of $l_{\mathrm{n}}$ cover the active region only. The resultant average saturation for these shadowed boxes is 
$1 \quad S_{b n} *=\left(S_{e}^{*}\right)\left(\frac{d_{f}}{D}\right)^{n^{*}}$

2 By definition, $S_{b n}$ should be equivalent to the effective water saturation $\left(S_{a}\right)$ within

3 the active region. Using $f$ to denote the fraction of the active region within the gridblock

4 and based on Equation (9), we have

$5 \quad f=\frac{S_{e}^{*}}{S_{a}}=\left(S_{e} *\right)^{\gamma}$

6 with

$7 \quad \gamma=1-\left(\frac{d_{f}}{D}\right)^{n^{*}}$

8 Parameter $\gamma$ is defined between zero and one. It is a key parameter for the active

9 region model. If flow pattern is uniform and does not contain preferential flow, $\gamma$ will be

10 equal to zero (corresponding to $d_{f}=D$ or $f=1$ ). Otherwise, $\gamma$ will be larger than zero and

11 result in an $f$ value less than one. In this case, only a portion of the flow domain

12 corresponding to preferential flow paths actually conducts liquid water. The $\gamma$ may be a

13 complex function of soil properties, flow conditions, and scale. In this study, we assume

14 it to be a constant for a given test site. (As will be discussed later in this paper, this

15 approximation seems to suffice for practical applications.) Liu et al. (2003) also

16 demonstrated that this approximation is valid for fingering flow in homogeneous porous

17 media.

$18 \quad 3.3$ Governing equations for water flow

As previously indicated, the active region model divides the flow domain into active and inactive regions. Flow occurs preferentially in the active region (characterized by 
1 fractals), with the inactive (immobile) region simply bypassed. The governing equation

2 for water flow can be expressed as (in the one-dimensional form):

$3 \quad \frac{\partial \theta}{\partial t}=\frac{\partial}{\partial z}\left[K\left(\frac{\partial h}{\partial z}+1\right)\right]-S$

4 where $\mathrm{z}$ is the vertical coordinate (positive upwards), $\mathrm{t}$ is time, $\mathrm{S}$ is the sink term, $\mathrm{h}$ is the

5 pressure head, $\theta$ is the total water content (including water contents from both active and

6 inactive regions), and $\mathrm{K}$ is the unsaturated hydraulic conductivity.

7 While the governing equation (12) is almost identical to the traditional continuum

8 approaches, the major difference between the active region model and the other models is

9 constitutive relations among saturation, capillary pressure, and unsaturated hydraulic

10 conductivity. To demonstrate this difference, consider the simplest case without

11 preferential flow and macropores. In such a case, the water capillary pressure head may

12 be described by the well-known van Genuchten relation (van Genuchten, 1980):

13

$$
h\left(S_{e}\right)=\frac{1}{\alpha}\left[S_{e}^{-1 / m}-1\right]^{1 / n}
$$

14

15 where $\alpha,\left(\mathrm{Pa}^{-1}\right), \mathrm{n},(-)$ and $\mathrm{m}=1-1 / \mathrm{n}$ are van Genuchten parameters. The effective 16 saturation is defined as

$17 S_{e}=\frac{\theta-\theta_{r}}{\theta_{s}-\theta_{r}}$

18 where $\theta_{\mathrm{s}}$ and $\theta_{\mathrm{r}}$ are saturated and residual water contents, respectively. 
1 In the active region model, however, the van Genuchten capillary-pressure relation is

2 considered to be relevant for the active region, rather than for the whole flow domain.

3 The capillary pressure for the active region is determined by replacing $S_{e}$ in Equation

4 (14) with $S_{a}$. From Equation (10), we obtain

5

6

7

8

9

10

11

12

13

14

15

16

17

18

19

$$
S_{a}=\left(S_{e}^{*}\right)^{1-\gamma}
$$

The saturation $\mathrm{S}_{\mathrm{e}}{ }^{*}$ can be related to total water saturation $\mathrm{S}_{\mathrm{e}}$ by

$$
S_{e}=f S_{a}+(1-f) S_{i}=S_{e}^{*}+\left(1-\left(S_{e}^{*}\right)^{\gamma}\right) S_{i}
$$

where $S_{i}$ is the water saturation within the inactive (immobile) region. It can be zero or a value corresponding to water flow that can be ignored compared to preferential flow (Larsson et al., 1999).

Equation (16) rather than (13) should be used to simulate water flow in soils with preferential flow. For a given effective water saturation, a larger $\gamma$ value corresponds to a larger effective water saturation $\left(S_{a}\right)$ within the active region, and therefore to a lower absolute value for capillary pressure (Liu et al., 1998).

The unsaturated hydraulic conductivity for the active region, $K_{a}$, is directly determined by the effective water saturation of the active region. However, because only a portion of the soil are active, the unsaturated hydraulic conductivity of the entire flow region (including both active and inactive regions), $K$, should be the $K_{a}$ multiplied by $f$, or 


$$
K=f K_{a}=\left(S_{e}^{*}\right)^{\gamma} K_{a}
$$

2 where $\mathrm{K}_{\mathrm{a}}$ can be given by the following van Genuchten permeability relation:

$3 \quad K_{a}=K_{s} S_{a}^{1 / 2}\left[1-\left\{1-S_{a}^{1 / m}\right\}^{m}\right]^{2}=K_{s}\left(S_{e}^{*}\right)^{(1-\gamma) / 2}\left[1-\left\{1-\left(S_{e}^{*}\right)^{(1-\gamma) / m}\right\}^{m}\right]^{2}$

4

where $\mathrm{Ks}$ is the saturated hydraulic conductivity.

5 Combining Equations (18) and (19) yields

$6 \quad K=K_{s}\left(S_{e^{*}}\right)^{(1+\gamma) / 2}\left[1-\left\{1-\left(S_{e}^{*}\right)^{(1-\gamma) / m}\right\}^{m}\right]^{2}$

7 In general, $\mathrm{K}$ is affected by $\gamma$ in a complex manner for a given $\mathrm{S}_{\mathrm{e}}$. A larger $\gamma$ value, 8 resulting in a higher effective water saturation within the active region $\left(S_{a}\right)$, gives rise to a

9 larger value of $\mathrm{K}_{\mathrm{a}}$. On the other hand, a larger $\gamma$ value corresponds to a smaller value of $\mathrm{f}$.

10 Because the former effect is dominant, a larger $\gamma$ value gives a larger $\mathrm{K}$ for a given

11 effective water saturation $\mathrm{S}_{\mathrm{e}}$ (Liu et al., 1998).

12 Note that the above discussion concerns water flow in a soil without macropores.

13 Therefore, a single set of van Genuchten parameters is used, but the active region model

14 is not limited to this special case. Two schemes can be used to include the effects of

15 macropores. One approach is to treat mcropore and soil matrix within the active region as

16 two coupled continua. Standard dual-continuum formulations can be borrowed here

17 (Simunek et al., 2003). The disadvantage of this approach is that additional parameters to

18 describe the coupling are needed. The other approach is to assume the local equilibrium

19 within the active region, considering that the high water saturation (and hydraulic

20 conductivity) generally existing within the active region could result in equilibrium

21 quickly. In this case, the composite constitutive relationships as described in Simunek et 
1 al. (2003) can be used to replace the van Genuchten relations in the above equations.

2 However, the reasonableness of this local equilibrium approximation needs to be further 3 evaluated.

4 It is instructive to compare the active region model with two models in the literature.

5 One of them is the active fracture model developed for simulating unsaturated flow and 6 transport in fractured rock (Liu et al., 1998, 2003). (We refer the readers to Liu et al. 7 (1998, 2003) regarding the details of the active fracture model and its validation results.)

8 Because both the active region model and active fracture model are based on the notion

9 that flow patterns in unsaturated systems are fractal, constitutive relationships for the two models are almost identical. Therefore, the active region model can be considered a direct extension of the active fracture model. However, it is useful to highlight the differences between the two models. First, because flow mechanisms are not exactly the same for unsaturated fractures and porous media (e.g., Liu and Bodvarsson, 2001), the two models are conceptually different. It is not appropriate to assume that a model valid for modeling flow in fractured rock will be equivalently valid for porous media. Furthermore, the active fracture model was developed to describe water flow and solute transport in fractures only, whereas our active region includes both soil matrix and macropores, as previously discussed. Also note that in the active fracture model, all the inactive fractures are assumed to have residual water saturation, whereas in the active region model, the immobile water saturation is not necessarily the same as the residual saturation. The similar treatment has been used in mobile/immobile models to be discussed later. As a result, the relationship between different water saturations in the active region model (Eq. 17) is more general than that in the active fracture model. 
The other model is the mobile/immobile model for simulating preferential flow in non-

2 structured soils (van Dam et al., 1990, 1996; Larsson et al., 1999). In this model, flow

3 domain is divided into mobile and immoble regions, corresponding to active and inactive

4 regions, respectively. van Dam et al. $(1990,1996)$ and Sexena et al. (1994) found that use

5 of the two-region model can significantly improve the comparisons between simulated

6 and observed results. However, in the mobile/immobile model, the fraction of the mobile

7 region (corresponding to our $\mathrm{f}$ in Equation (10)) was treated as constant. Both van Dam et

8 al. (1996) and Larsson et al. (1999) noticed that the fraction is not in reality a constant,

9 especially under the transient flow conditions. Van Dam et al. (1996) also called for

10 better practical concepts and models for simulating field-scale water flow and transport

11 (involving preferential flow) under transient conditions. Our active region can be

12 considered a generalization of the mobile/immobile model.

\section{A comparison with field observations}

The key element of the active region model is Equation (10). While the comprehensive evaluation of the active region model will be performed in our future research; we present our preliminary evaluation of Equation (10) in this study, based on data available in the literature.

Van Dam et al. (1990) used mobile/immobile model to analyze field tests involving application of a bromide solution to a field soil, subsequently leached by natural rainfall. The tests were carried out in two adjacent fields, one with a water-repellent top layer and one with a wettable top layer. Both soils are sandy soils. Using model calibration, van Dam et al. (1990) were able to estimate the fraction of mobile water (corresponding to $f$ in Equation (10) here) at three depth intervals for each of the two soils. Because, for a 
1 given depth, the water content did not change significantly during the tests, the

2 assumption of the constant fraction of mobile water for a given depth interval, used in

3 van Dam et al. (1990), is reasonable and not inconsistent with our Equation (10). As

4 shown in Fig 2, although the number of data points is limited for a given soil, the results

5 of van Dam et al. (1990) are fairly well represented by Equation (10) with different $\gamma$

6 values for the two different soils. The soil with the water-repellent top layer has a larger

$7 \gamma$ value (0.8) and therefore a larger degree of preferential flow, which is consistent with

8 findings in the literature (van Dam et al., 1990). Larsson et al. (1999) used the similar

9 mobile/immobile model to analyze test results for a sandy soil. Again, water content for a

10 given depth did not change significantly during tests. Fig. 2 also shows that the results of

11 Larsson et al. (1999) are consistent with Equation (10) with $\gamma=0.4$. Note that in the

studies of van Dam et al. (1990) and Larsson et al. (1999), macropores did not come to play in the observed flow process.

Recently, a number of studies of dye-stained preferential pathways have been published in the literature. Unfortunately, most of these studies did not include measurements of water content (or saturation) within the pathways, and therefore, cannot be used to directly evaluate Equation (10). One exception is the study reported by Öhrström et al. (2002). The tests were performed for six plots located in a clayey-soil site containing a considerable amount of macropores in the top layers. Soil samples were collected from an auger before and after tests for each plot. To compare the test results with Equation (10), we use the portion of dye coverage for a given depth as the portion of the active region. The observed water content is considered the content for the active region because it is higher than the initial water content observed before the tests started. 
1 Saturation within the active region is estimated from the observed water content. Because residual water content is not available, it is assumed to be zero. For a given depth, the average active water saturation $\left(\mathrm{S}_{\mathrm{e}}{ }^{*}\right)$ is estimated by the water saturation in the active region multiplied by the observed portion of dye coverage at that depth. Fig. 3 shows the data points at depths of 15, 50 and $75 \mathrm{~cm}$ for the six plots. As indicated in Öhrström et al. (2002), because water content values were observed from a single auger for a plot and water content may be highly variable, the observed water content data may only be indicative of actual soil water contents. Nevertheless, Fig 3 shows that the data points can be very well represented by our Equation (10) with $\gamma=0.8$.

The consistency between Equation (10) and the data points from different test sites

11 supports the usefulness of the active region model. Considering the relative simplicity of

12 the model and its capability for capturing fractal flow patterns, we believe that the active

13 region model can be used as a practical tool to deal with often-observed preferential flow

14 and transport. As expected, the $\gamma$ parameter is site specific (Figures 2 and 3). The 15 relationship of this parameter to other soil hydraulic parameters is important for practical 16 applications, but not clear at this stage. This issue needs to be investigated in future 17 research.

\section{Conclusions}

Preferential flow and transport are common processes in unsaturated soils. Development of theoretically rigorous and practically useful models is a current 21 challenge. Existing field evidence indicates that complex preferential flow patterns are fractals. The success of the developed discrete models in describing preferential flow at small scales is based on their ability to generate fractal patterns. In this study, we 
1 developed a relatively simple active region model to incorporate the fractal flow pattern

2 into the continuum approach. In the model, the flow domain is divided into active and

3 inactive regions. Flow occurs preferentially in the active region (characterized by

4 fractals) and inactive region is simply bypassed. Consistency between field observations

5 and the proposed model supports the usefulness of the model. However, further studies

6 are needed for more comprehensive evaluation of the active region model and for

7 developing relations between the $\gamma$ parameter (defined in Equation (10)) and the other soil

8 hydraulic parameters.

9

10

11

12

13

14

15

16

17

18

19

20

21

22

23 


\section{References}

2 Beven, K., Germann P., 1982. Macropores and water flow in soils. Water Resources

3 Research 18, 1311-1325.

4

5 Bouma, J., Dekker L.W., 1978. A case study on infiltration into dry clay soil, I.

6 Morphological observations. Geoderma 20, 27-40.

8 de Rooij, G.H., 2000. Modeling fingered flow of water in soils owing to wetting front 9 instability: a review. J. Hydrol. 231-232, 277-294.

10

11 Doughty, C., 1999. Investigation of conceptual and numerical approaches for evaluating

12 moisture, gas, chemical, and heat transport in fractured unsaturated rock. J. Contam.

13 Hydrol. 38, 69-106.

14

15 Ewing, R. P., Berkowitz. B., 2001. Stochastic pore-scale growth models of DNAPL 16 migration in porous media. Advances in Water Resources 24, 309-323.

17

18 Feder J. 1988. Fractals. Plenum Press, New York.

19 Flury, M., Fluhler H., Jury, W.A., Leuenberger, J., 1994. Susceptibility of soils to 20 preferential flow of water: A field study. Water Resources Research 30(7), 1945-1954. 
1 Flury M., Flühler H., 1995. Modeling solute leaching in soils by diffusion-limited 2 aggregation: Basic concepts and applications to conservative solutes. Water Resources 3 Research 31, 2443-2452.

4

5 Glass, R.J., Steenhuis T.S., Parlarge J.Y., 1988. Wetting front instability as a rapid and 6 far-reaching hydrologic process in the vadose zone. J. Contam. Hydrol. 3, 207-226.

8 Glass R.J. 1993. Modeling Gravity-Driven fingering using modified percolation theory. 9 In Proceedings of the fourth annual international conference on high level radioactive waste conference, Las Vegas, Nevada.

11

Hatano, R., Booltink H. W. G. 1992. Using fractal dimensions of stained flow patterns in a clay soil to predict bypass flow. J. Hydrol. 135, 121-131.

14

Hillel, D., 1987. Unstable flow in layered soils: A review. Hydrol. Processes 1, 143-147.

16

17 Larsson, M.H., Jarvis N.J., Torstensson G., Kasteel R., 1999. Quantifying the impact of 18 preferential flow on solute transport to tile drains in a sandy field soil. J. Hydrol. 215, 116-134.

20

Liu, H. H., Molz F.J., 1997. Multifractal analyses of hydraulic conductivity distribution. Water Resources Research 33, 2483-2488. 
1 Liu, H. H., Doughty, C., Bodvarsson G.S.. 1998. An active fracture model for unsaturated

2 flow and transport in fractured rocks. Water Resources Research 34, 2633-2646.

3

4 Liu, H.H., Zhang G., Bodvarsson G.S., 2003. The active fracture model: Its relation to

5 fractal flow behavior and a further evaluation using field observations, Vadose Zone

6 Journal (2): 259-269.

7

8 Liu, H.H., Bodvarsson G.S., 2001. Constitutive relations for unsaturated flow in a 9 fracture network, J. Hydrol. 252, 116-125.

10

11 Mandelbrot B. B. 1982. The fractal geometry of nature. W.H. Freeman, New York.

12

13 Meakin, P., Tolman S. 1989. Diffusion-limited aggregation. In Fractals in the natural sciences. Princeton Univ. Press, Princeton, N.J.

15

Molz, F.J., Boman G.K. 1993. A stochastic interpolation scheme in subsurface hydrology.

Water Resources Research 29, 3769-3774.

18

Molz, F.J.,Liu H.H., Szulga J. 1997. Fractional Brownian motion and fractional Gaussian noise in subsurface hydrology: A review, presentation of fundamental properties, and extensions. Water Resources Research 33, 2273-2286. 
1 Neuman, S.P. 1990. Uinversal scaling of hydraulic conductivities and dispersivities in 2 geologic media. Water Resources Research 26, 1749-1758.

3

4 Neuman, S.P. 1994. Generalized scaling of permeabilities: Validation and effect of support 5 scale. Geophys. Res. Lett. 21, 349-352.

6

7

8

9 Field-scale variation of preferential flow as indicated from dye coverage. J. Hydrol. 257, 10 164-173.

Öhrström P., Hamed Y., Persson M., Berndtsson R., 2004. Characterizing unsaturated solute transport by simultaneous use of dye and bromide. J. Hydrol. 289, 23-35.

Olsson, J., Persson M., Albergel J. Berndtsson R., Zante P., Ohrstrom P., Nasri S., 2002. Multiscaling analysis and random cascade modeling of dye infiltration. Water Resources Research 38(11), 1263. Doi:10.1029/2001WR00080.

Perfect, E., McLaughlin N.B., Kay B.D., Topp G.C.. 1996. An improved fractal equation for the soil water retention curve. Water Resources Research 32, 281-287.

Persson M., Yasuda H., Albergel H., Berndtsson R., Zante P., Nasri S., Ohrstrom P. 2001. Modeling plot scale dye penetration by a diffusion limited aggregation (DLA) model. J. Hydrol. 250, 98-105. 
2 Rieu, M., Sposito G., 1991a. Fractal fragmentation, soil porosity, and soil water

3 properties, 1. Theory. Soil Sci. Soc. Am. J. 55, 1231-1238.

4

5 Rieu, M., Sposito G. 1991b. Fractal fragmentation, soil porosity, and soil water 6 properties, 2. Applications. Soil Sci. Soc. Am. J., 55, 1231-1238.

8 Saxena, R.K., Jarvis N.J., Bergstrom L., 1994. Interpreting non-steady state tracer 9 breakthrough experiments in sand and clay soils using dual-porosity model. J Hydrol. $10 \quad 162,279-298$.

11

12 Smith J.E., Zhang Z. F. 2001. Determining effective interfacial tension and predicting 13 finger spacing for DNAPL penetration into water-saturated porous media. J. Contam. 14 Hydrol. 48, 167-183.

15

16 Simunek, J., Jarvis N.J., van Genuchten, M.Th., Gardenas A., 2003. Review and 17 comparison of models for describing non-equilibrium and preferential flow and transport 18 in the vadose zone. J. Contam. Hydrol. 272, 14-35.

19

20 Stauffer, D., Aharony, A. 1991. Introduction to Percolation Theory. Tayler \& Francis.

21 Tyler, S.W., Wheatcraft S.W., 1990. Fractal processes in soil water retention. Water 22 Resources Research 26, 1047-1054. 
1 van Dam, J.C., Hendrickx, J.H.M., van Ommen, H.C., Bannink M.H., van Genuchten M.

2 Th., Dekker L.W., 1990. Water and solute movement in a coarse-textured water-repellent

3 field soil. J. Hydrol. 120, 359-379.

4

5 van Dam, J.C., Wosten J.H.M., Nemes, A., 1996. Unsaturated soil water movement in 6 hysterestic and water repellent field soils. J. Hydrol. 184, 153-173.

8 van Genuchten, M. 1980. A closed-form equation for predicting the hydraulic 9 conductivity of unsaturated soil. Soil Sci. Soc. Amer. J. 44: 892-898.

10

11 Vogel, T., Gerke, H.H., Zhang R., van Genuchten M. Th. 2000. Modeling flow and 12 transport in a two-dimensional dual-permeability system with spatially variable hydraulic 13 properties. J. Hydrol. 238, 78-89.

14

15 Wang, Z., Wu L., Harter T., Lu J. Jury W.A., 2003. A field study of unsatuable 16 preferential flow during soil water redistribution. Water Resources Research 39(4), 1075, 17 doi:10.1029/2001WR000903.

18

19 Witten, T.A., Sander L.M. 1981. Diffusion-limited aggregation: A kinetic critical 20 phenomenon. Phys. Rev. Lett. 47, 1400-1403.

21

22 Wilkinson D., Willewsen J.F., 1983. Invasion percolation: A new form of percolation 23 theory. J. Phys. A. 16, 3365-3376. 
2 Yamamoto H., Kojima K., Tosaka H., 1993. Fractal clustering of rock fractures and its 3 modeling using cascade process. In Scale Effects in Rock Masses 93, Pinto da Cunha 4 (ed.). 1993 Balkema, Rotterdam.

5

6

7

8

9

10

11

12

13

14

15

16

17

18

19

20

21

22

23 


\section{$1 \quad$ Figures}

2

3 Fig. 1. Demonstration of the "box" counting procedure for several box sizes

4 Fig. 2 Comparison between Equation (10) and data from van Dam et al. (1990)

5 and Larsson et al. (1999). Note that Gamma here represents the factor $\gamma$ in Equation (10).

6 Fig. 3 A comparison between Equation (10) and data from Ohrstrom et al. (2002). Note

7 that Gamma here represents the factor $\gamma$ in Equation (10). 
. 
(a) $\mathrm{I}=\mathrm{L}$

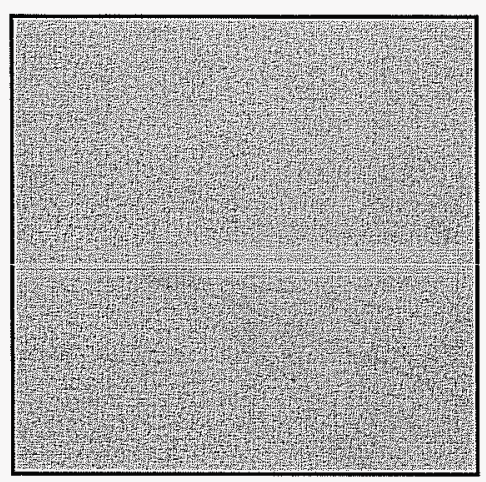

(c) $\mathrm{I}=\mathrm{L} / 4$

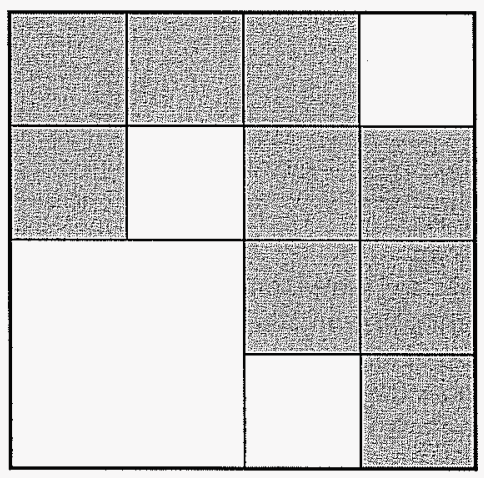

(b) $\mathrm{I}=\mathrm{L} / 2$

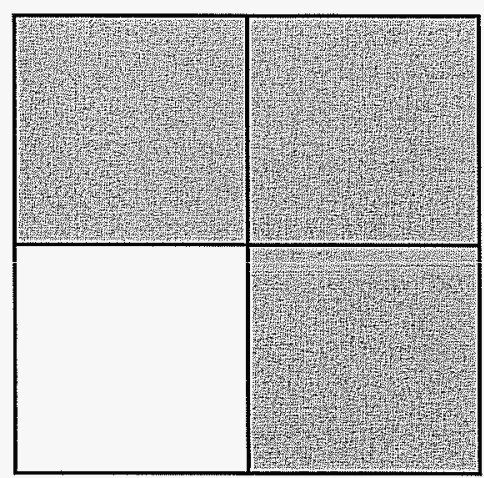

(d) $\mathrm{I}=\mathrm{L} / 8$

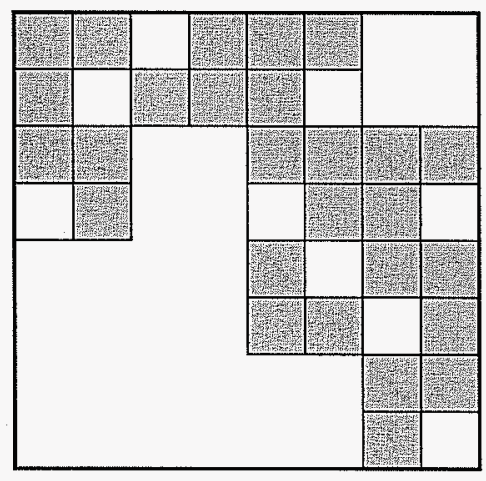

AT03-002

Fig. 1. Demonstration of the "box" counting procedure for several box sizes 


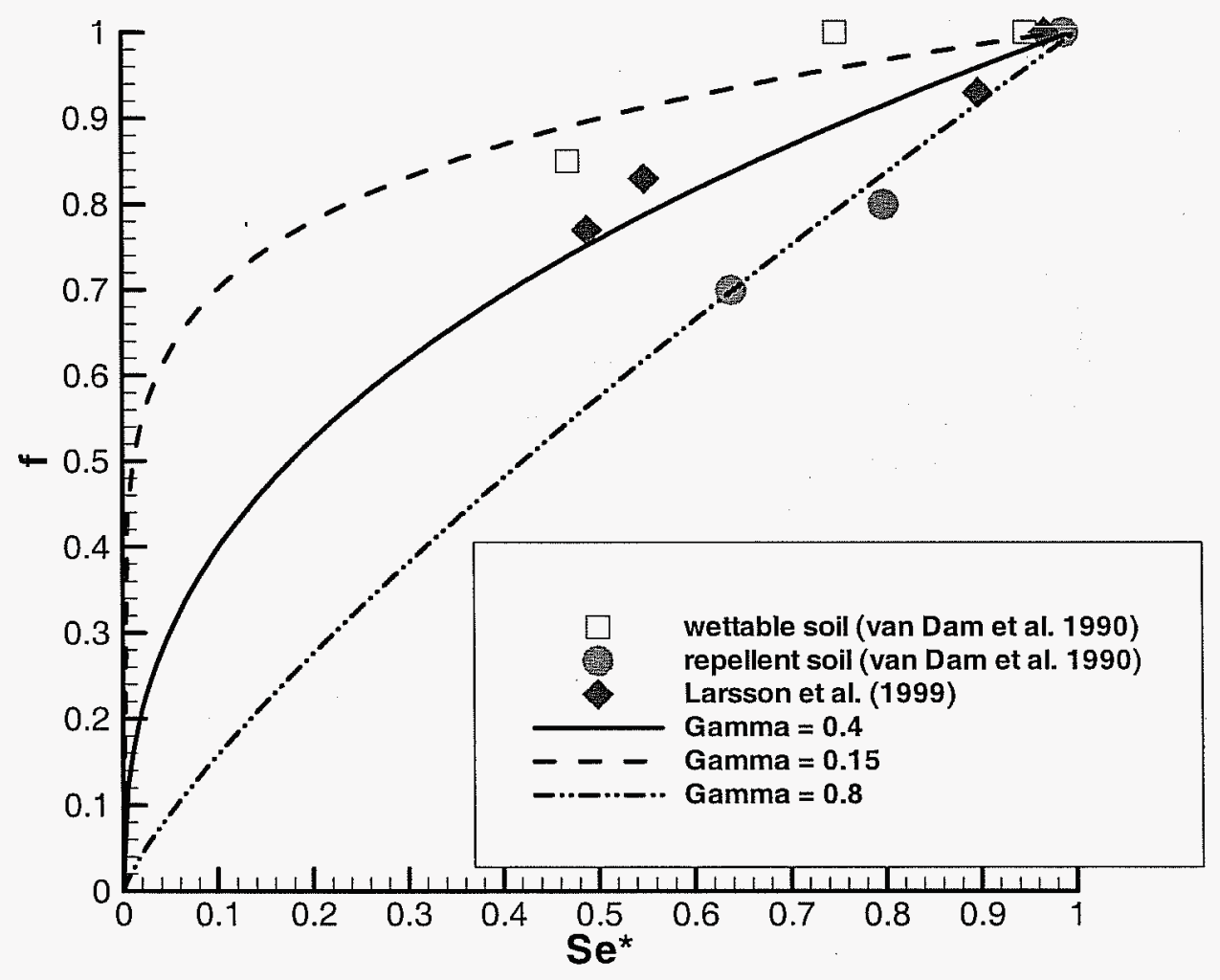

Fig. 2 Comparison between Equation (10) and data from van Dam et al. (1990) and Larsson et al. (1999) 


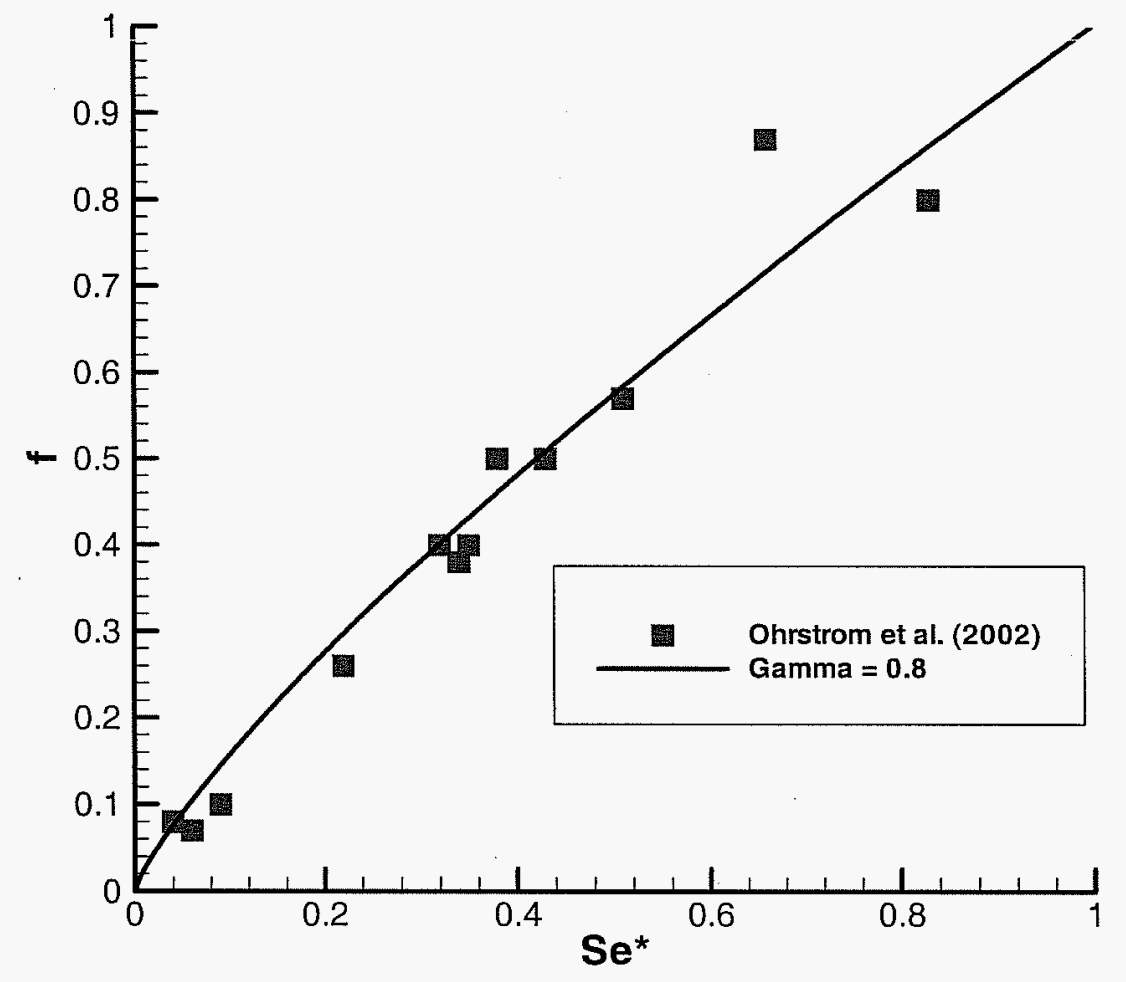

Fig. 3 A comparison between Equation (10) and data from Ohrstrom et al. (2002) 
• 